\title{
Preferences for Visual Stimuli Following Amygdala Damage
}

\author{
Ralph Adolphs and Daniel Tranel \\ University of Iowa College of Medicine
}

\begin{abstract}
- Bilateral damage to the human amygdala impairs retrieval of emotional and social information from faces. An important unanswered question concerns the specificity of the impairment for faces. To address this question, we examined preferences for a broad class of visual stimuli in two subjects with complete bilateral amygdala damage, both of whom were impaired in judgments of faces. Relative to controls, the subjects
\end{abstract}

showed a positive bias for simple nonsense figures, color patterns, three-dimensional-looking objects and landscapes. The impairment was most pronounced in regard to those stimuli that are normally liked the least. The human amygdala thus appears to play a general role in guiding preferences for visual stimuli that are normally judged to be aversive.

\section{INTRODUCTION}

A large body of work, primarily from studies in animals, has demonstrated the amygdala's importance in processing socially and emotionally relevant stimuli. Bilateral amygdala damage results in severe impairment in behaviors (and in the retrieval of knowledge) that normally are triggered by stimuli of direct importance to an animal's survival. The clearest demonstrations have come from paradigms in which the emotional significance of a stimulus can be experimentally manipulated, such as fear conditioning (Davis, 1992; Le Doux, 1996). The amygdala also plays a clear role in regard to stimuli encountered under ecologically more natural conditions: for instance, rats with bilateral amygdala damage show no fear of cats (Blanchard \& Blanchard, 1972), and monkeys with such damage are abnormally tame and placid in their interactions with people or with other monkeys (Amaral, Capitanio, Machado, Mason, \& Mendoza, 1997; Kling \& Brothers, 1992; Meunier, Bachevalier, Murray, Malkova, \& Mishkin, 1996; Rosvold, Mirsky, \& Pribram, 1954; Weiskrantz, 1956). Convergent neurophysiological data are available: Neurons within the primate amygdala respond to socially relevant stimuli, such as faces (Fried, MacDonald, \& Wilson, 1997; Rolls, 1992) and pictures of complex social interactions (Brothers, Ring, \& Kling, 1990).

Although impairments in real-life behavior following amygdala damage tend to be most apparent in the social domain (i.e., in regard to stimuli depicting, or behaviors directed at, other animals), there is evidence that the amygdala also plays a role in ecologically valid responses toward nonsocial stimuli. For instance, amygdalectomized monkeys will more readily approach unfamiliar

objects and appear less cautious in novel environments than normal monkeys (Amaral et al., 1997). Consonant with these findings, neurophysiological activity of neurons within the primate amygdala is modulated by the affective significance of a variety of nonsocial sensory stimuli (Muramoto, Ono, Nishijo, \& Fukuda, 1993; Nishijo, Ono, \& Nishino, 1988). All these data provide strong support for the view that the amygdala plays a critical role both in social behavior and in emotional response to nonsocial stimuli. However, nearly all available evidence has come from studies in nonhuman animals.

We have reported that bilateral amygdala damage also impairs social behavior in humans (Adolphs, Tranel, Damasio, \& Damasio, 1994; Tranel \& Hyman, 1990). In particular, such damage impairs judgments of the approachability and trustworthiness of other individuals on the basis of their facial appearance (Adolphs, Tranel, \& Damasio, 1998). Subjects with bilateral amygdala damage fail to judge people who look unapproachable and untrustworthy as such, while giving normal judgments to individuals who look approachable and trustworthy. The impairment appears to be limited to judging people from their faces and does not extend to judging people from lexical descriptions (Adolphs, Tranel, \& Damasio, 1998). Additionally, subjects with bilateral amygdala damage are impaired in recognizing certain negative emotions from facial expressions, notably fear and anger (Adolphs, Tranel, Damasio, \& Damasio, 1994; Adolphs, Tranel, Damasio, \& Damasio, 1995; Broks et al., 1998; Calder et al., 1996; Young et al., 1995). The findings from humans are thus broadly consistent with the findings from animals and support a role for the amygdala in 
triggering pertinent knowledge and behavior when subjects are confronted with social stimuli that signal potential threat or danger.

Studies of the human amygdala have predominantly found evidence for the amygdala's involvement in retrieving knowledge regarding faces. But this emphasis on faces could be artifactual: Few studies have used stimuli other than faces, and faces may also be the stimuli that most robustly trigger retrieval of social knowledge, due to the paramount role they play in real-life social behavior. Given that a few studies have implicated the human amygdala in processing auditory (Scott et al., 1997), olfactory (Zald \& Pardo, 1997), or gustatory (Zald, Lee, Fluegel, \& Pardo, 1998) emotional stimuli, we expected the amygdala to play a more general role in triggering emotional and social knowledge.

The present report thus addresses an important unanswered question: To what extent might the human amygdala participate in making emotionally relevant judgments regarding visual stimuli that are clearly not social? That is, might the above described impairments in judging faces extend to other classes of visual stimuli? To address this issue, we asked two subjects with complete bilateral amygdala damage (SM-046 and RH-1951; Figure 1) to rate how much they liked visual stimuli other than faces. Both subjects had previously been found to be severely impaired in similar judgments regarding faces (Adolphs et al., 1998). To obtain a broad assessment, we asked subjects to rate their preferences for four different classes of visual stimuli: unidentifiable black-and-white figures, Mondrian color patterns, colored spheres, and colored landscapes (Figure 2). Performances from the two subjects with bilateral amygdala damage were compared to a group of young and a group of older normal controls.

\section{RESULTS}

The two groups of normal controls did not differ in their ratings of the four classes of stimuli, as confirmed by a one-way multivariate analysis of variance (MANOVA) on subjects' mean ratings (Wilks Lambda $=0.94 ; F(4)=1.29$; $p=0.28$ ). For statistical comparisons with data from amygdala subjects, we therefore pooled the data from the two control groups.

We divided the ratings that subjects assigned to the stimuli in two, one half contained the stimuli to which control subjects gave the most negative ratings and the other contained those to which controls gave the most positive ratings. Both SM-046 and RH-1951 gave higher ratings to the stimuli normally given the most negative ratings (Figure 3 ). The results were especially striking for the nonsense line drawings and spheres: SM-046 and $\mathrm{RH}-1951$ gave mean positive ratings to these stimuli even when mean control ratings were negative. By contrast, the mean ratings that SM-046 and RH-1951 assigned to stimuli that received the most positive ratings from normal controls did not differ from the control group (Figure 3). Statistical analysis of these data showed that amygdala subjects were significantly impaired in rating that half of the nonsense figures and spheres that normally receive the most negative ratings $(t=-15.3 ; p<$ 0.001 for line drawings and $t=-10.0 ; p<0.05$ for spheres) but not those that normally receive the most positive ratings ( $p$ s $>0.4$; one-tailed $t$ tests on subjects mean ratings for the given classes of stimuli, with $p$ values Bonferroni-corrected for multiple comparisons). Due to the small number of stimuli and the large variance in ratings given by subjects (including controls), statistical significance was not attained for the data from Mondrians or landscapes $(p s>0.1)$. Nonetheless, subjects with bilateral amygdala damage gave both Mondri-

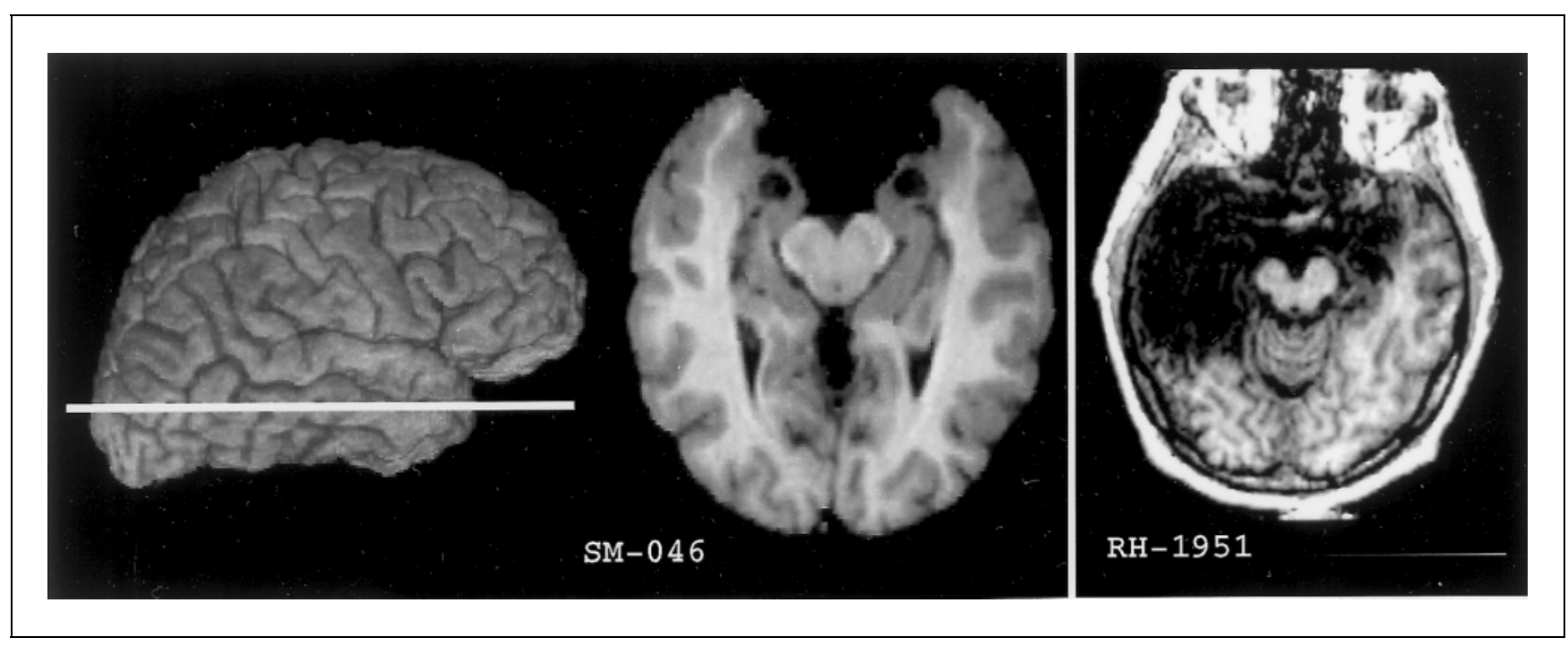

Figure 1. Bilateral amygdala lesions as seen in magnetic resonance scans of the brains of SM-046 (left) and RH-1951 (right). Shown on the far left is a three-dimensional reconstruction of SM-046's brain from MR scans indicating the plane of section for the other images. SM-046 has bilateral damage restricted to the amygdala; RH-1951 has extensive bilateral damage to amygdala, hippocampus, and surrounding cortices. 


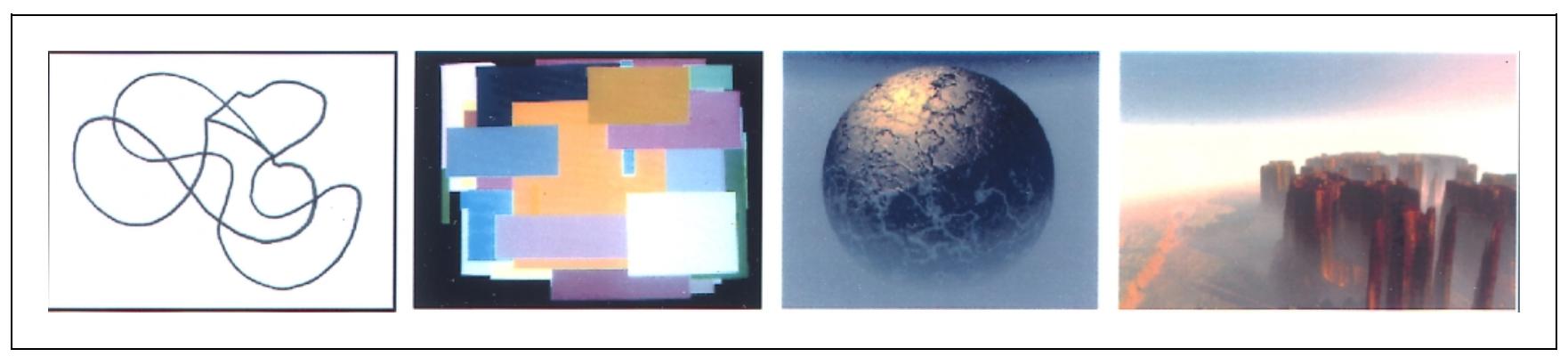

Figure 2. Examples of stimuli used. From left to right: nonsense line figure, Mondrian, sphere, landscape.

ans and landscapes more positive ratings than the mean control rating, for that half of the stimuli normally receiving the most negative ratings (Figure 3 ).

The data of Figure 3 suggest that subjects with bilateral amygdala lesions gave the most abnormal ratings to those stimuli that normally receive the most negative ratings. An analysis of the extent to which SM-046 and RH-1951's ratings deviated from the control mean for each individual stimulus confirmed this impression (Figure 4): The most abnormal ratings were given to those stimuli that were rated the most negatively by controls. Regressions of the deviations of ratings given by amygdala subjects on the rank-order of the ratings given to stimuli by normal controls revealed statistical significance in several cases, as indicated in the figure. Moreover, all regressions were in the same direction for the four different classes of stimuli: Subjects with amygdala damage gave abnormally positive ratings to stimuli for which normal controls gave the most negative ratings. This pattern of performance was found previously in subjects with bilateral amygdala damage, in regard to judging social attributes of faces (Adolphs et al., 1998).

\section{DISCUSSION}

\section{Summary of Findings}

The findings from this study show that bilateral amygdala damage in humans results in abnormally positive ratings of likability for several classes of visual stimuli other than faces. The data show a close parallel to those reported in regard to social judgments of faces (Adolphs et al., 1998): Subjects with amygdala damage endorse positive ratings for stimuli that normal subjects rate negatively. As with social judgment of faces, this positive bias was most pronounced in regard to those stimuli that normal subjects gave the most negative likability ratings. The human amygdala thus appears to play a general role in guiding preferences for visual stimuli that are normally judged to be aversive or to predict aversive consequences. This function may be especially critical, or may be most perspicuous, in regard to judgment of social stimuli such as faces.

Although the data clearly show a positive bias by subjects with bilateral amygdala damage, and although the deviation of the amygdala subjects' ratings from normal ratings was greatest for those stimuli that normally receive the most negative ratings, we cannot definitively conclude that the impairment is restricted to judging negative (disliked, aversive) stimuli. In fact, an inspection of SM-046's data in Figure 3 shows that, like normals, she generally gave "likable" stimuli more positive ratings than "dislikable" stimuli. It thus remains possible that the disproportionate impairment seen for the most dislikable stimuli is due to a general positive bias, together with a ceiling effect introduced by our bounded rating scale. It might be the case that it is simply possible to deviate more in the positive direction for the dislikable stimuli than for the likable stimuli. Future experiments involving direct pairwise comparisons between stimuli (such as have been carried out in regard to faces; Adolphs et al., 1998) would be needed to exclude this possibility.

The established role of the human amygdala in recognizing facial expressions of fear, its role in recognizing untrustworthiness and avoidance of faces, and the present evidence for a role in recognizing dislike in nonsocial stimuli all make plausible the following hypothesis. Stimuli that have been associated with negative (aversive) consequences in the past (possibly either in individual experience or through phylogeny) activate the amygdala to trigger responses such that the organism can avoid the aversive consequence that is predicted by the stimulus. This hypothesis is consonant with a large body of data from studies in animals, using both the lesion method and direct neurophysiological recording (Davis, 1992; Le Doux, 1996; Schoenbaum, Chiba, \& Gallagher, 1998). The amygdala may trigger behavioral reaction, conscious knowledge that the stimulus predicts something "bad," or a feeling that one does not like, or wishes to avoid, the stimulus.

\section{Factors Contributing to Preference Judgments}

A difficult question concerns how stimuli in the task are categorized as similar to previously encountered stimuli that coincided with negative contingencies or consequences. What precisely is it about, say, an unidentifiable 


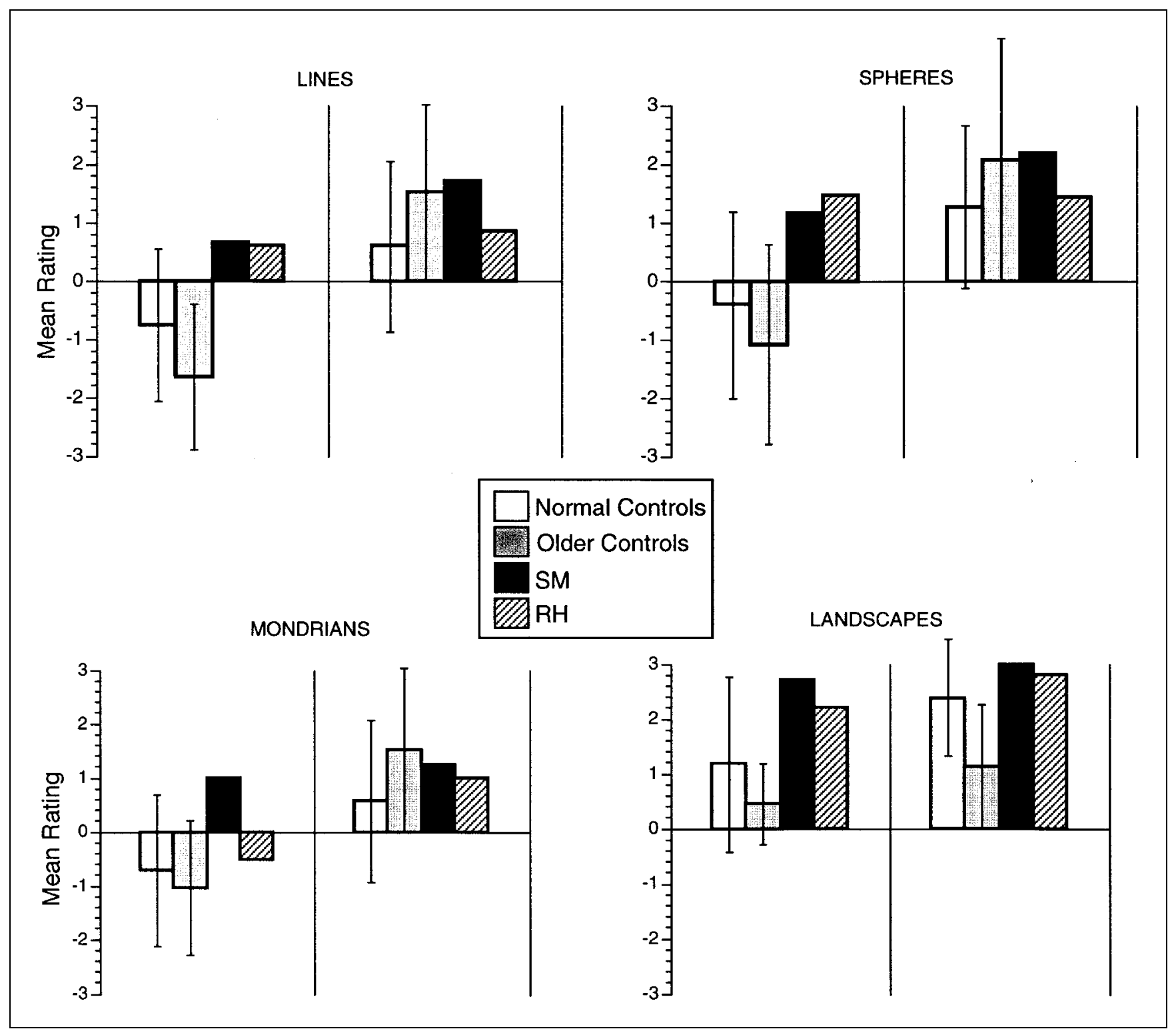

Figure 3. Mean ratings given by subjects to that half of the stimuli that normal controls assign the most negative ratings (left balf of each split graph) and to that half of the stimuli that normal controls assign the most positive ratings (right balf of each split graph). Means and standard deviations are shown for young normal controls and older normal controls; individual mean ratings are shown for SM-046 and RH1951.

nonsense figure or a colored sphere that makes a subject like or dislike the item? Although there is some debate in the literature, complexity (Wohlwill, 1968), amplitude spectra (Kleiner, 1987), prior exposure (Kunst-Wilson \& Zajonc, 1980), and averageness (Langlois \& Roggman, 1990), among other factors, have all been proposed to influence preferences for visual stimuli. It will be a challenge for future studies to begin to dissect the relative contributions these factors make to judging stimuli such as those we used here and to investigate their possible dependency on the amygdala.

A related issue concerns the connection between likability judgments and the retrieval of emotionally relevant knowledge about the stimuli, on which such judg- ments may in part be based. It is important to note that the amygdala is not necessary for all types of judgments regarding potentially aversive stimuli. For instance, an amnesic patient with complete bilateral amygdala damage was found to have normal covert preferences for other people: He could judge which people had been kind to him in the past and which had not (his amnesia precluded overt judgments; Tranel \& Damasio, 1993). Additionally, patients with bilateral amygdala damage are not impaired in their personal emotional judgments of complex visual stimuli that show recognizably emotional objects or scenes (e.g., Adolphs, Cahill, Schul, \& Babinsky, 1997). However, the stimuli used in that study differed importantly from the ones used in the present study, in 


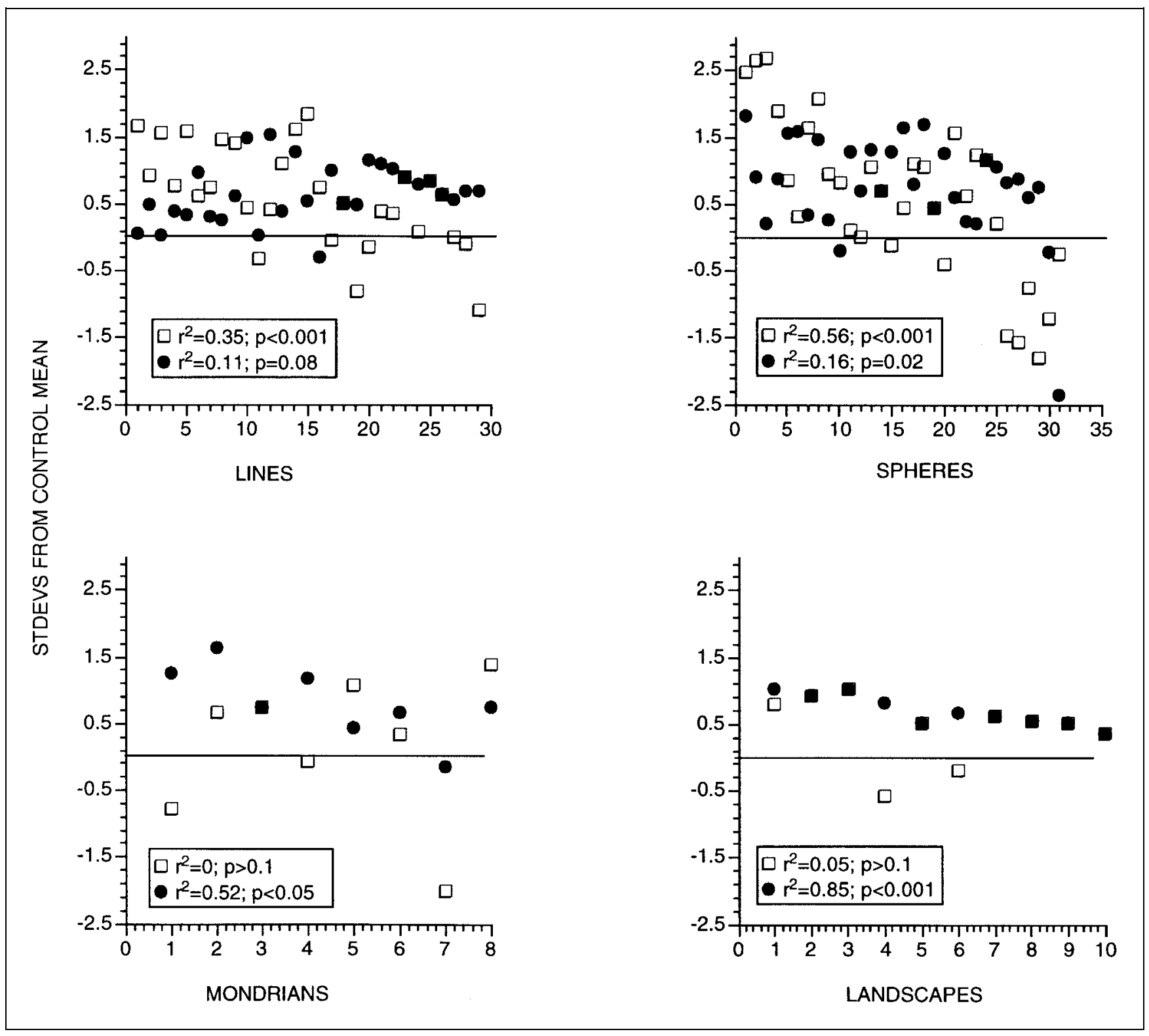

Figure 4. Standard deviations from the mean of the normal controls for RH-1951 ( $\square$ ) and SM-046 (O). Stimuli are rank-ordered on the $x$-axis according to the mean ratings that normal controls (young and old collapsed) gave them. For each dataset, we regressed the amygdala subject's deviation against the normal controls' rank order ( $y$ value against $x$ value); $r^{2}$ and $p$ values resulting from this regression are indicated on the graphs.

that recognizing the stimulus (e.g., picture of a car accident or of a mutilated person) could trigger sufficient declarative knowledge on the basis of which a normal emotional judgment could be made. Such explicit pointers toward emotionally relevant information may not be triggered by the stimuli we used here, which cannot be recognized as obviously depicting an emotionally aversive situation or event.

Perhaps for similar reasons, subjects with bilateral amygdala damage are not impaired in judging recognizable scenes or objects with respect to pleasantness or unpleasantness (Hamann, Cahill, \& Squire, 1997; Adolphs,
Russell, \& Tranel, 1999). Interestingly, a subject with bilateral amygdala damage was impaired in recognizing the emotional arousal of certain classes of unpleasant stimuli, even though she recognized their unpleasantness normally (Adolphs et al., 1999). All the above findings argue that there are multiple mechanisms for the retrieval of knowledge regarding various attributes of emotional stimuli. The amygdala plays a critical role only for a subset of these, which may be a function of the mode of knowledge retrieval (overt/covert, motor response/verbal response/autonomic response, etc.) and of the type of knowledge retrieved (e.g., knowledge of 
pleasantness, arousal, or the specific emotion category; knowledge that can be encoded verbally or not; and so on). The extent to which each of these contributes to judgments of likability as assessed in the present study remains to be fully determined.

It would be of interest also to extend this line of investigation to other classes of stimuli. Important issues would include an examination of judgments of social visual stimuli other than faces (e.g., body posture) and of social and nonsocial stimuli in sensory modalities other than vision. Another interesting question concerns the relative contributions of the amygdala to preferences that are innate and to preferences that are acquired through experience. Either, or both, innate and acquired preferences could account for the data we presented here: Subjects may like a specific stimulus because they have an innate bias for the stimulus or because the stimulus is associated with a particular prior experience. Examination of subjects who acquired their amygdala damage at different ages could provide insight into this difficult issue and connect with the extensive literature on emotional and social development in normal individuals (Saarni, Mumme, \& Campos, 1997).

\section{METHODS}

\section{Subjects}

We examined two subjects with bilateral amygdala damage, SM-046 (a 31-year-old woman) and RH-1951 (a 42year-old man). Both subjects have been described previously, have normal visuoperceptual function, and are severely impaired in processing social (Adolphs et al., 1998) and emotional (Adolphs et al., 1994, and unpublished observations) information from faces. Their performances were compared to those given by 114 normal control subjects (or a subset thereof, as indicated below). Because the mean age of the normal controls (college students; mean age $=19 \pm 1$ ) was younger than that of our brain-damaged subjects, we tested an additional 8 older normal subjects ( 4 males, 4 females; $59 \pm$ 12 years old). All subjects had given informed consent to participate in these studies.

Both SM-046 and RH-1951 have complete bilateral damage to the amygdala. SM-046's lesions are confined to the amygdala, with the exception of minimal damage to the anterior entorhinal cortex. The lesions are a consequence of mineralization and atrophy of the amygdala due to Urbach-Wiethe disease. RH-1951's lesions are due to herpes simplex encephalitis at age 28 , which resulted in complete destruction of the amygdala and surrounding structures. We obtained detailed neuroanatomical information on SM-046 and RH-1951 from serial magnetic resonance (MR) scans suitable for subsequent three-dimensional reconstruction of subjects' brains (Damasio \& Frank, 1992) (Figure 1).

\section{Stimuli}

Individual stimuli were unfamiliar to all subjects, to avoid the influence of a subject's personal experience with a specific stimulus. We investigated stimuli that derive from classes of items that subjects generally have some familiarity with (landscapes, planets) and stimuli that derive from classes of items that subjects likely have never seen before (nonsense figures, Mondrian patterns).

We used four types of stimuli: simple unidentifiable black-and-white nonsense line drawings (drawn on a white background), arrangements of colored rectangles (Mondrians), colored spheres that had been rendered to look three-dimensional (resembling planets in appearance), and computer-generated landscapes. Examples of each type of stimulus are shown in Figure 2. All stimuli were shown one at a time in random order, without time limit, on a backward-projection screen from a slide projector in a darkened room. Subjects were asked to rate how much they liked each stimulus, on a scale of -3 to +3 . After a subject rated the stimulus, there was a 3-sec interstimulus-interval showing a black screen before the next stimulus came on. Negative ratings corresponded to dislike of the stimulus; positive ratings corresponded to liking the stimulus. The number of normal control subjects was 85 for the unrecognizable figures, 111 for spheres, and 114 for Mondrians and landscapes.

Stimuli were selected from a larger set, to be evenly distributed between very disliked (mean control rating near -3 ) to very liked (mean control rating near +3 ) by normal subjects.

Unidentifiable line drawings. Twenty-nine unidentifiable black nonsense figures were shown on a plain white background.

Mondrians. Eight images of Mondrians were chosen from a larger set. All were vividly colored collages of rectangles.

Spheres. Thirty-one images of a three-dimensional sphere with colored patterns on it were computer generated. These often resembled planets in appearance (cf. Figure 2). Some fog and directional lighting were used to enhance the three-dimensional effect.

Landscapes. Ten images of landscapes were computer generated using the program KPT Bryce. They showed three-dimensional renditions of terrain in which the topography, lighting, clouds, and fog had been manipulated with the aim to elicit strong positive or negative emotional feelings in viewers.

\section{Acknowledgments}

We thank Antonio R. Damasio for helpful comments on the manuscript, J. Suhr for technical assistance in testing subjects, and D. Krutzfeldt for help with scheduling subjects. This study was supported in part by an NIH FIRST Award and an Alfred P. 
Sloan Research Fellowship to R.A. and by a grant from the National Institute for Neurological Diseases and Stroke to Antonio R. Damasio.

Reprint requests should be sent to Ralph Adolphs, Dept. of Neurology, 200 Hawkins Drive, Iowa City, IA 52242.

\section{REFERENCES}

Adolphs, R., Cahill, L., Schul, R., \& Babinsky, R. (1997). Impaired declarative memory for emotional material following bilateral amygdala damage in humans. Learning and Memory, 4, 291-300.

Adolphs, R., Russell, J. A., \& Tranel, D. (1999). A role for the human amygdala in recognizing emotional arousal. Psychological Science, 10, 167-171.

Adolphs, R., Tranel, D., \& Damasio, A. R. (1998). The human amygdala in social judgment. Nature, 393, 470-474.

Adolphs, R., Tranel, D., Damasio, H., \& Damasio, A. (1994). Impaired recognition of emotion in facial expressions following bilateral damage to the human amygdala. Nature, 372, 669-672.

Adolphs, R., Tranel, D., Damasio, H., \& Damasio, A. R. (1995). Fear and the human amygdala. Journal of Neuroscience, $15,5879-5892$

Amaral, D. G., Capitanio, J. P., Machado, C. J., Mason, W. A., \& Mendoza, S. P. (1997). The role of the amygdaloid complex in rhesus monkey social behavior. Society for Neuroscience Abstracts, 23, 570.

Blanchard, D. C., \& Blanchard, R. J. (1972). Innate and conditioned reactions to threat in rats with amygdaloid lesions. Journal of Computational Physiology \& Psychology, 81, 281-290.

Broks, P., Young, A. W., Maratos, E. J., Coffey, P. J., Calder, A. J., Isaac, C., Mayes, A. R., Hodges, J. R., Montaldi, D., Cezayirli, E., Roberts, N., \& Hadley, D. (1998). Face processing impairments after encephalitis: Amygdala damage and recognition of fear. Neuropsychologia, 36, 59-70.

Brothers, L., Ring, B., \& Kling, A. (1990). Response of neurons in the macaque amygdala to complex social stimuli. Behavioral Brain Research, 41, 199-213.

Calder, A. J., Young, A. W., Rowland, D., Perrett, D. I., Hodges, J. R., \& Etcoff, N. L. (1996). Facial emotion recognition after bilateral amygdala damage: Differentially severe impairment of fear. Cognitive Neuropsychology, 13, 699-745.

Damasio, H., \& Frank, R. (1992). Three-dimensional in vivo mapping of brain lesions in humans. Archives of Neurology, 49, 137-143.

Davis, M. (1992). The role of the amygdala in conditioned fear. In J. P. Aggleton (Ed.), The amygdala: Neurobiological aspects of emotion, memory, and mental dysfunction. New York: Wiley-Liss.

Fried, I., MacDonald, K. A., \& Wilson, C. L. (1997). Single neuron activity in human hippocampus and amygdala during recognition of faces and objects. Neuron, 18, 753-765.

Hamann, S. B., Cahill, L., \& Squire, L. R. (1997). Emotional perception and memory in amnesia. Neuropsychology, 11, 104-113.

Kleiner, K. A. (1987). Amplitude and phase spectra as indices of infant's pattern preferences. Infant Bebavior and Development, 10, 49-59.

Kling, A. S., \& Brothers, L. A. (1992). The amygdala and social behavior. In J. P. Aggleton (Ed.), The amygdala: Neurobi- ological aspects of emotion, memory, and mental dysfunction. New York: Wiley-Liss.

Kunst-Wilson, M. R., \& Zajonc, R. (1980). Affective discrimination of stimuli that cannot be recognized. Science 207, 557-558.

Langlois, J. H., \& Roggman, L. A. (1990). Attractive faces are only average. Psychological Science, 1, 115-121.

Le Doux, J. (1996). The emotional brain. New York: Simon \& Schuster.

Meunier, M., Bachevalier, J., Murray, E. A., Malkova, L., \& Mishkin, M. (1996). Effects of aspiration vs. neurotoxic lesions of the amygdala on emotional reactivity in rhesus monkeys. Society for Neuroscience Abstracts, 22, 1867.

Muramoto, K., Ono, T., Nishijo, H., \& Fukuda, M. (1993). Rat amygdaloid neuron responses during auditory discrimination. Neuroscience, 52, 621-636.

Nishijo, H., Ono, T., \& Nishino, H. (1988). Single neuron responses in amygdala of alert monkey during complex sensory stimulation with affective significance. Journal of Neuroscience, 8, 3570-3583.

Rolls, E. T. (1992). Neurophysiology and functions of the primate amygdala. In J. P. Aggleton (Ed.), The amygdala: Neurobiological aspects of emotion, memory, and mental dysfunction (pp. 143-167). New York: Wiley.

Rosvold, H. E., Mirsky, A. F., \& Pribram, K. (1954). Influence of amygdalectomy on social behavior in monkeys. Journal of Computational Physiology \& Psychology, 47, 173-178.

Saarni, C., Mumme, D. L., \& Campos, J. J. (1997). Emotional development: Action, communication, and understanding. In W. Damon (Ed.), Handbook of child psychology: Social, emotional, and personality development (vol. 3, pp. 237309). New York: Wiley.

Schoenbaum, G., Chiba, A. A., \& Gallagher, M. (1998). Orbitofrontal cortex and basolateral amygdala encode expected outcomes during learning. Nature Neuroscience, 1, 155-159.

Scott, S. K., Young, A. W., Calder, A. J., Hellawell, D. J., Aggleton, J. P., \& Johnson, M. (1997). Impaired auditory recognition of fear and anger following bilateral amygdala lesions. $\mathrm{Na}$ ture, 385, 254-257.

Tranel, D., \& Damasio, A. R. (1993). The covert learning of affective valence does not require structures in hippocampal system or amygdala. Journal of Cognitive Neuroscience, 5, 79-88.

Tranel, D., \& Hyman, B. T. (1990). Neuropsychological correlates of bilateral amygdala damage. Archives of Neurology, 47, 349-355.

Weiskrantz, L. (1956). Behavioral changes associated with ablation of the amygdaloid complex in monkeys. Journal of Computational Physiology \& Psychology, 49, 381-391.

Wohlwill, J. F. (1968). Amount of stimulus exploration and preference as differential functions of stimulus complexity. Perception and Psychophysics, 4, 307-312.

Young, A. W., Aggleton, J. P., Hellawell, D. J., Johnson, M., Broks, P., \& Hanley, J. R. (1995). Face processing impairments after amygdalotomy. Brain, 118, 15-24.

Zald, D. H., Lee, J. T., Fluegel, K. W., \& Pardo, J. V. (1998). Aversive gustatory stimulation activates limbic circuits in humans. Brain, 121, 1143-1154.

Zald, D. H., \& Pardo, J. V. (1997). Emotion, olfaction, and the human amygdala: Amygdala activation during aversive olfactory stimulation. Proceedings of the National Academy of Sciences, USA, 94, 4119-4124. 